\title{
The Peierls - Nabarro Model of Dislocations: A Venerable Theory and its Current Development
}

Chapter · January 2005

DOI: 10.1007/978-1-4020-3286-8_41

CITATIONS

42

1 author:

\section{Gang Lu}

California State University, Northridge

142 PUBLICATIONS $\quad 2,253$ CITATIONS

SEE PROFILE
READS

95 


\title{
THE PEIERLS-NABARRO MODEL OF DISLOCATIONS: A VENERABLE THEORY AND ITS CURRENT DEVELOPMENT
}

\author{
Gang Lu \\ Division of Engineering and Applied Science, Harvard University, \\ Cambridge, Massachusetts, USA
}

Dislocations are central to the understanding of mechanical properties of crystalline solids. While continuum elasticity theory describes well the long-range elastic strain of a dislocation for length scales beyond a few lattice spacings, it breaks down near the singularity in the region surrounding the dislocation center, known as the dislocation core. There has been a great deal of interest in describing accurately the dislocation core structure on an atomic scale because of its important role in many phenomena of crystal plasticity [1-3]. The core properties control, for instance, the mobility of dislocations, which accounts for the intrinsic ductility or brittleness of solids. The core is also responsible for the interaction of dislocations at close distances, which is relevant to plastic deformation.

Two types of theoretical approaches have been employed to study dislocation core properties. The first is based on direct atomistic simulations using either empirical interatomic potentials or ab initio calculations. Empirical potentials involve the fitting of parameters to a predetermined database and hence may not be reliable in predicting the core properties, where severe distortions like bond breaking, bond formation and switching necessitate a quantum mechanical description of the electronic degrees of freedom. On the other hand, ab initio total energy calculations, though considerably more accurate, are computationally expensive for the studies of dislocation properties. The second approach is based on the framework of the Peierls-Nabarro $(\mathrm{P}-\mathrm{N})$ model which holds the promise of becoming a plausible alternative to direct atomistic simulations. For this reason, there has been a resurgence of interest in the simple and tractable $\mathrm{P}-\mathrm{N}$ model for studying the dislocation core structure and mobility. In particular, the $\mathrm{P}-\mathrm{N}$ model permits easy estimation of the key dislocation

S. Yip (ed.),

Handbook of Materials Modeling. Volume I: Methods and Models, 1-19.

(C) 2005 Springer. Printed in the Netherlands. 
characteristics of nucleation and mobility, directly from quantities (GSF energy, see later) accessible through standard quantum mechanical or empirical atomistic computations.

\section{Original P-N Model}

Peierls [4] first proposed the remarkable hybrid model in which some of the details of the discrete dislocation core were incorporated into an essentially continuum framework. Nabarro [5] and Eshelby [6] further developed Peierls' model and gave the first meaningful estimate of the lattice friction to dislocation motion. Later attempts to generalize the original treatment of Peierls and Nabarro assumed a more general core configuration from which they derived the interactions across the glide plane which satisfy the Peierls integral equation. The basic idea of the P-N model can be illustrated in Fig. 1. The dislocated solid is separated into two elastic half-spaces joined by atomic-level forces across their common interface, known as the glide plane. The dislocation is characterized by a slip distribution $\delta(x)=\mathbf{u}\left(x, 0^{+}\right)-\mathbf{u}\left(x, 0^{-}\right)$, where $\mathbf{u}(x)$ is the displacement vector at position $x$ in the glide plane. The goal of the $\mathrm{P}-\mathrm{N}$ model is to determine the slip distribution $\delta(x)$ (or the displacement field $\mathbf{u}(x)$ ) across the glide plane that minimizes the total energy of the solid. The total energy includes two distinct contributions that compete with each

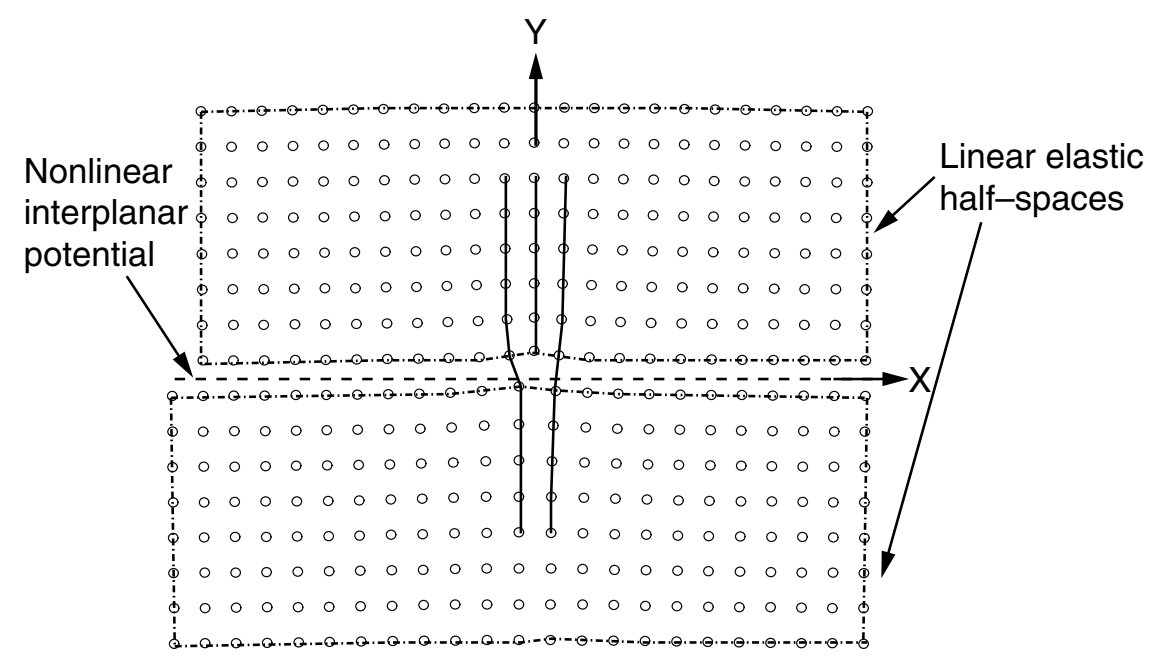

Figure 1. A schematic illustration showing an edge dislocation in a lattice. The partition of the dislocated lattice into linear elastic region and nonlinear atomistic region allows a multiscale treatment of the problem. 
other in determining the equilibrium slip distribution. One of contributions accounts for the atomic interaction across the glide plane which reflects the fact that there is an energy penalty for the misfit across the glide plane. Such misfit energy can be written as

$$
U_{\text {misfit }}=\int_{-\infty}^{+\infty} \gamma(\delta(x)) \mathrm{d} x,
$$

where $\gamma(\delta(x))$ is the generalized stacking fault (GSF) energy defined as the following [7]: consider a perfect crystal cut across a single plane into two parts which are then subjected to a relative displacement through an arbitrary vector $\delta$ and rejoined. The reconnected lattice has a surplus energy per unit area $\gamma(\delta)$. As the vector $\delta$ is varied to span a unit cell of the interface, $\gamma(\delta)$ generates the generalized stacking fault energy surface. The procedure can be repeated for various crystal planes. The significance of the GSF energy surface (or $\gamma$-surface) is that for a fault vector $\delta$ there is an interfacial restoring stress

$$
\mathbf{F}_{b}(\boldsymbol{\delta})=-\nabla(\gamma(\boldsymbol{\delta}))
$$

which has the same formal interpretation as the restoring stress in the $\mathrm{P}-\mathrm{N}$ model. Note that the GSF energy surface retains the translational and rotational symmetry of the underlying lattice. For example, there is no attendant energy cost if the atoms across the glide plane experience a relative displacement equal to the Burgers vector.

The second energy contribution to the total energy is the elastic energy stored in the two elastic half-spaces. This energy corresponds to the elastic energy of the dislocation, and it depends on the slip distribution $\delta(x)$ as well. Without losing generality, we can assume a one-dimensional slip of $\delta(x)$ first, and deal with a three-dimensional slip $\delta(x)$ later. As pointed out by Eshely [6], a straight dislocation can be represented as a continuous distribution of infinitesimal dislocations whose Burgers vectors are defined as the local gradient of the slip distribution. For example, the infinitesimal dislocation lying between $x^{\prime}$ and $x^{\prime}+\mathrm{d} x^{\prime}$ has a Burgers vector

$$
\mathrm{d} b\left(x^{\prime}\right)=\left(\frac{\mathrm{d} \delta(x)}{\mathrm{d} x}\right)_{x=x^{\prime}} \mathrm{d} x^{\prime} \equiv \rho\left(x^{\prime}\right) \mathrm{d} x^{\prime},
$$

where the local slip gradient $\rho(x)$ is also called dislocation density. Integrating the dislocation density over all $x$ we find

$$
\int_{-\infty}^{+\infty} \rho(x) \mathrm{d} x=\int_{-\infty}^{+\infty} \frac{\mathrm{d} \delta(x)}{\mathrm{d} x} \mathrm{~d} x=\delta(+\infty)-\delta(-\infty)=b,
$$

which is what we would expect from the definition of the dislocation density (see Fig. 1). These infinitesimal dislocations interact elastically, and the total 
elastic energy can be obtained through the superposition principle by adding up the contribution from each infinitesimal dislocation separately. More specifically, an infinitesimal edge dislocation located at $x^{\prime}$ produces a shear stress at some other point $x$ which is given by

$$
\sigma_{x y}(x, 0)=K_{e} \frac{\mathrm{d} b\left(x^{\prime}\right)}{x-x^{\prime}},
$$

$K_{e}$ is the prelogarithmic elastic factor for an edge dislocation. The displacement $u(x)$ necessary to create the infinitesimal dislocation at $x$ takes place in the presence of the shear stress from the dislocation at $x^{\prime}$, giving the following contribution to the elastic energy from the latter dislocation:

$$
\mathrm{d} U_{\text {elastic }}=K_{e} \frac{d b\left(x^{\prime}\right)}{x-x^{\prime}} u(x) .
$$

Integrating this expression over all values of $x$ from $-L$ to $L$, and over $\mathrm{d} b\left(x^{\prime}\right)$ to add the contribution from all infinitesimal dislocations, we obtain the total elastic energy of the original dislocation

$$
U_{\text {elastic }}=K_{e} \int_{-L}^{L} \int_{0}^{b} u(x) \frac{\mathrm{d} b\left(x^{\prime}\right)}{x-x^{\prime}} \mathrm{d} x=K_{e} \int_{-L}^{L} \int_{-L}^{L} u(x) \frac{\rho\left(x^{\prime}\right) \mathrm{d} x^{\prime}}{x-x^{\prime}} \mathrm{d} x,
$$

where $L$ is an inconsequential constant introduced as a large cutoff distance. Performing an integration by parts over $x$, we arrive the following expression for the elastic energy:

$$
U_{\text {elastic }}=K_{e} b^{2} \ln L-\frac{K_{e}}{2} \int_{-L}^{L} \int_{-L}^{L} \rho(x) \rho\left(x^{\prime}\right) \ln \left|x-x^{\prime}\right| \mathrm{d} x \mathrm{~d} x^{\prime} .
$$

Similar results can be also found for a screw dislocation with $K_{e}$ replaced by the corresponding elastic constant for the screw dislocation. For a general mixed dislocation with an angle $\theta$ between the dislocation line and its Burgers vector, the elastic energy is given by

$$
U_{\text {elastic }}=K b^{2} \ln L-\frac{K}{2} \int_{-L}^{L} \int_{-L}^{L} \rho(x) \rho\left(x^{\prime}\right) \ln \left|x-x^{\prime}\right| \mathrm{d} x \mathrm{~d} x^{\prime},
$$

where

$$
K=\frac{\mu}{2 \pi}\left(\frac{\sin ^{2} \theta}{1-v}+\cos ^{2} \theta\right),
$$

for an isotropic solid. $\mu$ and $v$ are the shear modulus and Poisson's ratio, respectively. This result clearly separates the contribution of the long-range 
elastic field of the dislocation, embodied in the first term of Eq. (9), from the contribution of the large distortions at the dislocation core, embodied in the second term of the equation. We will drop the first term in our later discussion and concentrate on the second term, which represents the energy contribution from the dislocation core.

Now we arrive expression of the total energy of the dislocation as a functional of dislocation density $\rho(x)$ :

$$
U_{t o t}[\rho(x)]=\int_{-\infty}^{+\infty} \gamma(\delta(x)) \mathrm{d} x-\frac{K}{2} \int_{-L}^{L} \int_{-L}^{L} \rho(x) \rho\left(x^{\prime}\right) \ln \left|x-x^{\prime}\right| \mathrm{d} x \mathrm{~d} x^{\prime} .
$$

By minimizing the above energy functional, we can find the equilibrium structure of the dislocation. A variational derivative of Eq. (11) with respect to the dislocation density $\rho(x)$ leads to the $\mathrm{P}-\mathrm{N}$ integro-differential equation:

$$
K \int_{-\infty}^{+\infty} \frac{1}{x-x^{\prime}} \frac{\mathrm{d} \delta\left(x^{\prime}\right)}{\mathrm{d} x^{\prime}} \mathrm{d} x^{\prime}=F_{b}(\delta(x))
$$

If a simple sinusoidal form is assumed for $F_{b}(\delta(x))$, as in the original P-N treatment, the misfit is then given by the well-known analytical solution,

$$
\delta(x)=\frac{b}{\pi} \tan ^{-1} \frac{x}{\zeta}+\frac{b}{2},
$$

where

$$
\zeta=\frac{K b}{4 \pi F_{\max }}=\frac{\mathrm{d}}{2(1-v)}
$$

is the half-width of the dislocation core and $F_{\max }=\mu b /(2 \pi d)$ is the maximum restoring stress with $d$ as the interlayer distance between the glide planes. One of the key features that emerges from this solution is that the $\mathrm{P}-\mathrm{N}$ model removes the artificial divergence at the core that is associated with the idealized continuum dislocation of Volterra. By introducing the nonlinear and nonconvex interplanar potential into the model, the solution of $\mathrm{P}-\mathrm{N}$ model in terms of stress and strain is seen to be well behaved.

One of the achievements of the $\mathrm{P}-\mathrm{N}$ model is that it provides a reasonable estimate of the dislocation size, characterized by $\zeta$ as a result of the competition between the two energy contributions. The more important achievement of the $\mathrm{P}-\mathrm{N}$ model is that it offers an insight into the value of the critical stress to move a dislocation in an otherwise perfect lattice. Such stress has thus been termed as Peierls stress. In order to derive the Peierls stress, however, the $\mathrm{P}-\mathrm{N}$ energy functional needs to be modified. The expression in Eq. (11) that we have discussed so far is invariant with respect to an arbitrary translation of the dislocation density $\rho(x) \rightarrow \rho(x+t)$. In other words, the dislocation 
described by the $\mathrm{P}-\mathrm{N}$ solution does not experience any resistance as it moves through the lattice. This is clearly unrealistic, and is a consequence of neglecting the discrete nature of the lattice: The $\mathrm{P}-\mathrm{N}$ model views the solid as a continuous medium. The only effect of the lattice periodicity. so far, comes from the periodicity of the misfit potential with a period of the Burgers vector. In order to rectify this problem and to recover the lattice resistance of dislocation motion, the $\mathrm{P}-\mathrm{N}$ energy functional was modified so that the misfit potential is not sampled continuously as in Eq. (11), but only at the positions of the actual atomic planes. This amounts to the following modification of the first term in total energy of the dislocation in Eq. (11):

$$
\int_{-\infty}^{+\infty} \gamma(\delta(x)) \mathrm{d} x \rightarrow \sum_{n=-\infty}^{+\infty} \gamma\left(\delta\left(x_{n}\right)\right) \Delta x,
$$

where $x_{n}$ is the position of the $n$th atomic planes and $\Delta x$ is the spacing between these atomic planes. Assuming a sinusoidal restoring stress $F[\delta(x)]=$ $\mu b /(2 \pi d) \sin [2 \pi \delta(x) / b]$, the misfit potential (Frenkel potential) $\gamma[\delta(x)]$ is

$$
\gamma[\delta(x)]=\frac{F_{\max } b}{2 \pi}\left[1-\cos \frac{2 \pi \delta(x)}{b}\right] .
$$

If the center of the dislocation is displaced by $\alpha b$ with $\alpha<1$, the total misfit energy becomes:

$$
U_{\text {misfit }}=\frac{\mu b^{3}}{8 \pi^{2} \mathrm{~d}} \sum_{n=-\infty}^{+\infty}\left[1+\cos 2\left\{\tan ^{-1} 2(1-v)\left(\alpha+\frac{n}{2}\right) b / \mathrm{d}\right\}\right],
$$

where we have used Eq. (13) for $\delta(x)$, and Eq. (16) for $\gamma(\delta)$ to evaluate the misfit energy in Eq. (15). After appropriate manipulations, Eq. (17) may be rewritten as

$$
U_{\text {misfit }}(\alpha)=\frac{\mu b^{3}}{4 \pi^{2} \mathrm{~d}} \frac{4 \zeta^{2}}{b^{2}} \sum_{n=-\infty}^{+\infty} \frac{1}{(2 \zeta / b)^{2}+(2 \alpha+n)^{2}},
$$

which can then be handled by the Poisson summation formula

$$
\sum_{n=-\infty}^{+\infty} f(n)=\sum_{k=-\infty}^{+\infty} \int_{-\infty}^{+\infty} f(x) e^{2 \pi \mathrm{i} k x} \mathrm{~d} x
$$

After performing the relevant integrations, we arrive the final expression for the misfit energy

$$
U_{\text {misfit }}(\alpha)=\frac{\mu b^{2}}{4 \pi(1-v)}+\frac{\mu b^{2}}{2 \pi(1-v)} \exp \left(\frac{-4 \pi \zeta}{b}\right) \cos 4 \pi \alpha .
$$

From the above expression, we see that the straight dislocation experiences a periodic potential wells and in the act of passing from one potential well 
to the next it must cross an energy barrier known as the Peierls barrier $W_{p}$. The stress required to surmount this energy barrier is the Peierls stress, $\sigma_{p}$, given by [8]

$$
\begin{aligned}
\sigma_{p} & =\frac{1}{b^{2}}\left[\frac{\partial U_{\text {misfit }}(\alpha)}{\partial \alpha}\right]_{\max } \equiv \frac{2 \pi W_{p}}{b^{2}} \\
& =\frac{2 \mu}{1-v} \exp \left(-\frac{4 \pi \zeta}{b}\right)=\frac{2 \mu}{1-v} \exp \left(-\frac{2 \pi d}{b(1-v)}\right) .
\end{aligned}
$$

A few observations are now in place. First, we note that the Peierls stress is extremely sensitive to the ratio of $(\zeta / b)$ or $(d / b)$ for fixed values of elastic constants $\mu$ and $\nu$. Therefore an edge dislocation is more mobile than a screw dislocation with the same Burgers vector in the same material since the edge dislocation is wider (larger $\zeta$ ) than the corresponding screw dislocation. In general, the more the edge component of a mixed dislocation, the wider the dislocation core, and hence the greater the mobility. In a given crystal, the slip system of dislocations corresponds to the largest value of $(d / b)$, namely, the slip plane tends to have the largest interplanar spacing, and the slip direction or Burgers vector is along the nearest neighbor direction (smaller $b$ ). In closepacked metallic systems, the values of $(\zeta / b)$ and $(d / b)$ are large, and these materials are usually ductile. In contrast, crystals with more complex unit cells (such as ceramics) have relatively small $d / b$ ratio, giving larger Peierls stress. In these materials, the shear stress for dislocation motion cannot be overcome before fracturing the solid, thus they are usually brittle. A second observation to be made concerns the magnitude of the Peierls stress. What we find from the $\mathrm{P}-\mathrm{N}$ model is that the stress to move a dislocation is down by an exponential factor in comparison with the shear modulus. It explains the fact that in many materials, plastic deformation operates at a shear stress that is orders of magnitude below its shear modulus: it is all due to dislocation motion! A final observation that we should make is, that dislocation motion often takes place by kink mechanisms in which a bulge on the dislocation line brings a segment of the dislocation into adjacent Peierls valleys, and the resulting kinks propagate with the end result being a net forward motion of the dislocation. In this case, what the kinks have to overcome is the secondary Peierls barrier along the dislocation line direction.

Despite the great heuristic value and useful insight that the original $\mathrm{P}-\mathrm{N}$ model offers, it lacks the quantitative power for prediction. In particular, the model becomes increasingly inaccurate for dislocations with narrow cores, as is typically the case in covalently bonded solids. Since the P-N model represents a combination of the continuum model and the GSF interplanar potential, its accuracy can be affected by either component. One of the main deficiencies of the original $\mathrm{P}-\mathrm{N}$ model is the assumption of sinusoidal force law. In real materials, however, the interplanar potential (GSF energy) is not 
at all sinusoidal. At present, the GSF energies can be calculated very accurately by using an $a b$ initio quantum mechanical framework, which brings to the problem of possible inaccuracies in the continuum component. In the following, we will address the limitations in the original $\mathrm{P}-\mathrm{N}$ model, and present some solutions that have been put forward to improve the quantitative description of dislocation core properties and mobility.

(1) The original P-N model is based on the isotropic or the pseudo-isotropic elasticity theory. Recently, full anisotropic treatments have been implemented in the $\mathrm{P}-\mathrm{N}$ model, which do not require much more computational effort [9].

(2) The original P-N model is one-dimensional, assuming the slip is along one direction. This "constrained path approximation" fails for dislocations in many Bravais lattices. For example, in an fcc lattice, dislocations can dissociate into partials whose Burgers vectors are not parallel, and a treatment in two dimension is mandatory. Currently, many $\mathrm{P}-\mathrm{N}$ theories have been proposed to solve this problem. For example, we will introduce a powerful $\mathrm{P}-\mathrm{N}$ model, namely, the Semidiscrete Variational P-N (SVPN) model which is capable to deal with three-dimensional displacement field, particularly useful for studying narrow dislocations.

(3) The original P-N model yields a variation of the misfit energy and the Peierls stress which has a periodicity of $b / 2$, in contrast with the feature of the dislocation barrier, which must in general, exhibit the periodicity of the Burgers vector $b$. There have been controversies and confusions with regard to this problem (e.g., see [10]), and it was attributed to an erroneous representation of the atomic positions across the glide plane in the original $\mathrm{P}-\mathrm{N}$ model after the dislocation is translated by a distance [11]. By correcting this error, one can recover the correct periodicity of $b$ for the misfit energy and the Peierls stress. Another relevant idea has also been exploited in a numerical formulation of the $\mathrm{P}-\mathrm{N}$ model. Namely, the misfit energy is not summed over the the position of atomic nuclei, but rather it is averaged over the Thomas-Fermi radius around the nuclei. This modification is particularly useful for metallic systems where electrons are more delocalized. It has been shown that the Peierls barrier and Peierls stress can be lowered considerably by this modification [12].

(4) In the original P-N model, the Peierls stress is derived by considering the misfit energy exclusively (see Eq. (20)). The elastic energy is assumed to be constant, in other words, the dislocation shape is assumed to be rigid during the dislocation translation process. This assumption turns out to be unrealistic. In fact, it is critical to include the variation of the elastic energy in Eq. (20) when evaluating the Peierls stress. However in doing so, one faces an inconsistency in the formulation: while the elastic energy is computed by a continuous integration, the misfit energy has to be sampled discretely in order to incorporate the discrete nature of lattice. Thus, the two energy contributions are not treated on the equal footing and the total energy is not variational. The 
SVPN model was developed precisely to resolve this inconsistency by discretizing the elastic energy [13]. As we shall see later, the variational formulation of the $\mathrm{P}-\mathrm{N}$ theory permits us to compute the Peierls stress more accurately by allowing the dislocation shape to change during the translation. The relaxation of dislocation core is particularly important for narrow dislocations. In fact, it has been shown that the Peierls stress can be reduced by three orders of magnitude for the screw dislocation in Si by allowing the relaxation of the dislocation shape. The reduced Peierls stress is in a much better agreement with the direct atomistic simulation result. We should emphasize that the $\mathrm{P}-\mathrm{N}$ model calculation takes only a small fraction of the computational time (a few minutes) that a direct atomistic simulation may take (hours or even days).

\section{Semidiscrete Variational P-N Model}

In the remaining of this article, we will introduce the SVPN model to exemplify the current development of the $\mathrm{P}-\mathrm{N}$ theory. There are two versions (planar and nonplanar) of the SVPN model that have been developed. The planar model aims to treat a dislocation that is entirely confined to a single glide plane, while the nonplanar model deals with a dislocation that is spread onto more than one glide planes. The nonplanar model was developed in order to study stress-assisted dislocation cross-slip and constriction [14]. After the discussion of the models, we will apply the planar model to dislocations in $\mathrm{Al}$ with ab initio determined GSF energy surface.

To facilitate the presentation, we adopt the following conventions. As defined in Fig. 2, xoz plane is the (111) glide plane for Al, $z$ axis is in the direction of the dislocation line, and $x$ axis is the glide direction, with $y$ axis normal to the glide plane. For planar dislocations, the displacements along $y$ direction are usually small. The Burgers vector $\mathbf{b}$ lies on the glide plane making an angle $\theta$ with the $z$ axis. The Burgers vector is along $x$ axis $\left(\theta=90^{\circ}\right)$ for an edge dislocation and along $z$ axis $\left(\theta=0^{\circ}\right)$ for a screw dislocation. The Burgers vector of a mixed dislocation has both an edge component, $b \sin \theta$, and a screw component, $b \cos \theta$. In general, the atomic displacements have components in all three directions rather than only along the direction of the Burgers vector, because the path along the Burgers vector may have to surmount a higher interplanar energy barrier in the GSF surface (see Fig. 4).

In the planar SVPN formalism, the dislocation slip is assumed to be confined to a single glide plane, separating two semi-infinite linear elastic continua. The equilibrium structure of a dislocation is obtained by minimizing the dislocation energy functional [15]

$$
U_{\text {disl }}=U_{\text {elastic }}+U_{\text {misfit }}+U_{\text {stress }}+K b^{2} \ln (L),
$$




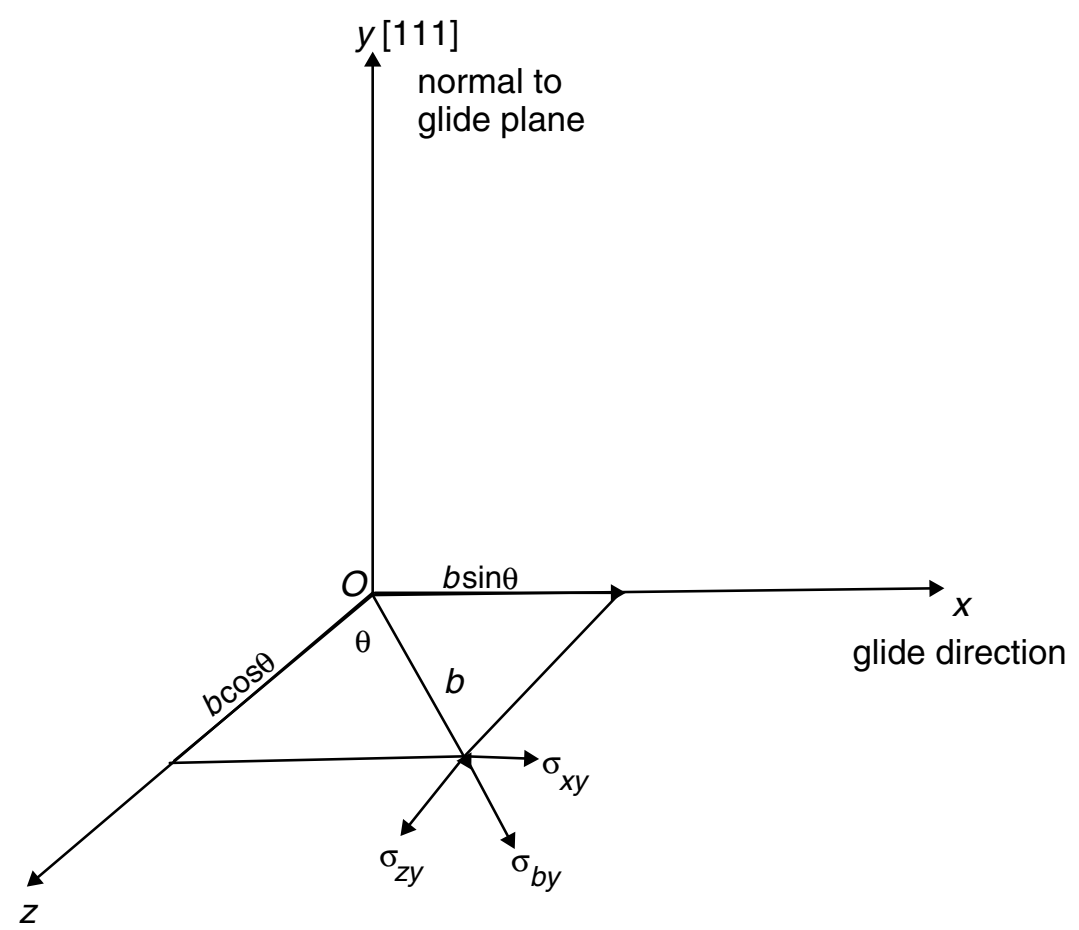

dislocation line

Figure 2. Cartesian set of coordinates showing the directions relevant for dislocations in $\mathrm{Al}$.

where

$$
\begin{aligned}
& U_{\text {elastic }}[\{\rho\}]=\sum_{i, j} \frac{1}{4 \pi} \chi_{i j}\left[K_{e}\left(\rho_{i}^{(1)} \rho_{j}^{(1)}+\rho_{i}^{(2)} \rho_{j}^{(2)}\right)+K_{s} \rho_{i}^{(3)} \rho_{j}^{(3)}\right], \\
& U_{\text {misfit }}[\{\delta\}]=\sum_{i} \Delta x \gamma_{3}\left(\delta_{i}\right), \\
& U_{\text {stress }}[\{\rho\}, \tau]=-\sum_{i, l} \frac{x_{i}^{2}-x_{i-1}^{2}}{2}\left(\rho_{i}^{(l)} \tau_{i}^{(l)}\right),
\end{aligned}
$$

with respect to the dislocation density or the slip vector. Here, $\rho_{i}^{(1)}, \rho_{i}^{(2)}$, and $\rho_{i}^{(3)}$ are the edge, vertical and screw components of the general dislocation density at the $i$ th nodal point and $\gamma_{3}\left(\delta_{i}\right)$ is the three-dimensional GSF energy surface. $\Delta x$ is the area assigned to each atomic row (the length of all dislocation lines is $1 \AA$ ). The corresponding components of the applied stress interacting with the $\rho_{i}^{(1)}, \rho_{i}^{(2)}$, and $\rho_{i}^{(3)}$, are $\tau^{(1)}=\sigma_{21}, \tau^{(2)}=\sigma_{22}$ and 
$\tau^{(3)}=\sigma_{23}$, respectively. $K, K_{e}$, and $K_{s}$ are the prelogarithmic energy factors defined earlier. The dislocation density at the $i$ th nodal point is defined as $\rho_{i}=\left(\delta_{i}-\delta_{i-1}\right) /\left(x_{i}-x_{i-1}\right)$. The remaining quantities entering in this expression are: $\chi_{i j}=\frac{3}{2} \phi_{i, i-1} \phi_{j, j-1}+\psi_{i-1, j-1}+\psi_{i, j}-\psi_{i, j-1}-\psi_{j, i-1}$, with $\phi_{i, j}=x_{i}-x_{j}$, and $\psi_{i, j}=\frac{1}{2} \phi_{i, j}^{2} \ln \left|\phi_{i, j}\right|$.

The first term in the energy functional, $U_{\text {elastic }}$, represents the configurationdependent (density or slip) part of the elastic energy, which has been discretized. Since any details of the displacements across the glide plane other than those on the atomic rows are disregarded, it is consistent to assume that the dislocation density is constant between the nodal points. This explicit discretization of the elastic energy term removes the inconsistency in the original $\mathrm{P}-\mathrm{N}$ model and produces a total energy functional which is variational. Another modification in this approach is that the nonlinear misfit potential in the energy functional, $U_{\text {misfit }}$, is a function of all three components of the nodal displacements, $\delta\left(x_{i}\right)$. Namely, in addition to the displacements along the Burgers vector, lateral and even vertical displacements across the glide plane are also included. This in turn, allows the treatment of straight dislocations of arbitrary orientation in arbitrary glide planes. Furthermore, because the slip vector $\delta\left(x_{i}\right)$ is allowed to change during the process of dislocation translation, the Peierls energy barrier can be significantly lowered compared to its corresponding value from a rigid translation. In order to examine the trend of energetics for different dislocations, we identify the dislocation configurationdependent part of the total energy as the core energy, $U_{\text {core }}=U_{\text {elastic }}+U_{\text {misfit }}$, which includes the density-dependent part of the elastic energy and the entire misfit energy, in the absence of external stress. The last term in Eq. (22), $K b^{2} \ln (L)$, is independent of the dislocation density, and hence, is irrelevant in the variational procedure and has no contribution to the evaluation of the Peierls stress (a typical value for the outer cutoff radius $L$ is $10^{3} \AA$; we use this value for all dislocations in the calculations discussed below).

The response of a dislocation to an applied stress is determined by the minimization of the energy functional with respect to $\rho_{i}$ at a given value of applied stress, $\tau_{i}^{(l)}$. An instability is reached when an optimal solution for $\rho_{i}$ no longer exists, which is manifested numerically by the failure of the minimization procedure to convergence. The Peierls stress is defined as the critical value of the applied stress which gives rise to this instability.

Having developed the planar SVPN model, it is not difficult to extend it to more than one glide plane. We have recently developed the nonplanar SVPN model in order to study dislocation cross-slip and constriction in fcc metals [14]. As shown in Fig. 3, a screw dislocation placed at the intersection of the primary (plane I) and cross-slip plane (plane II) is allowed to spread into the two planes simultaneously. The $X\left(X^{\prime}\right)$ axis represents the glide direction of the dislocation at the plane I (II). For an fcc lattice, the two slip 


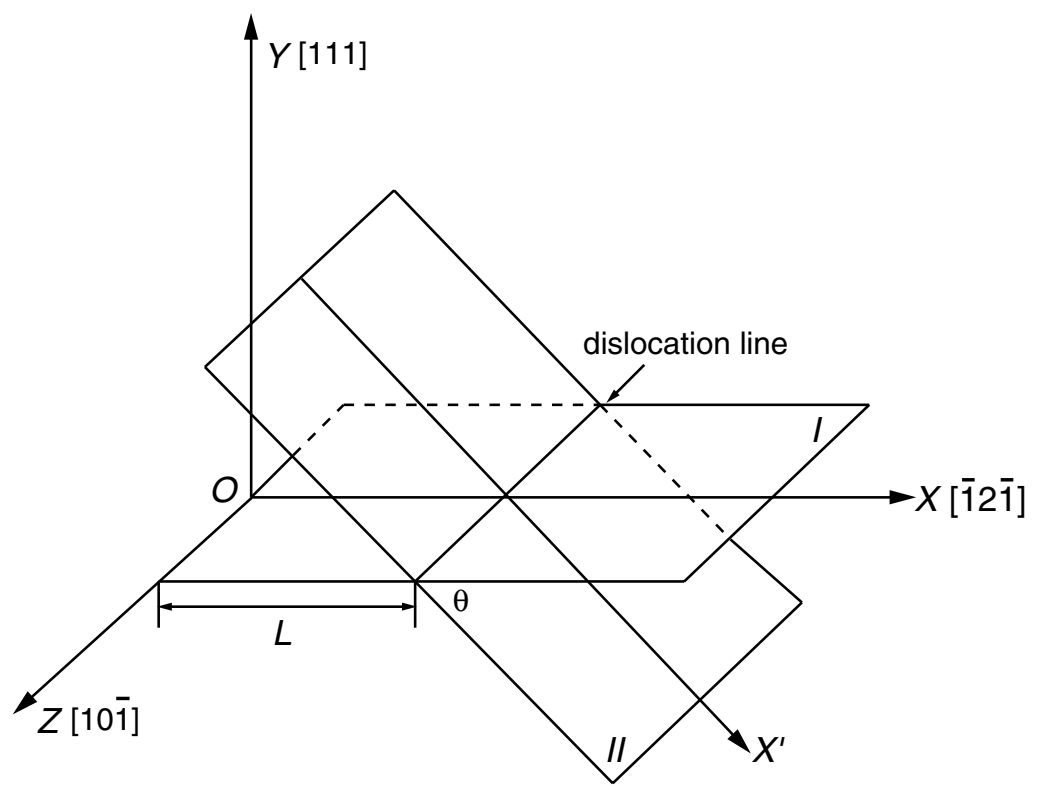

Figure 3. Cartesian set of coordinates showing the directions relevant to the screw dislocation located at the intersection of the two glide planes. Plane I (II) denotes the primary (cross-slip) plane.

planes are (111) and (111), forming an angle $\theta \approx 71^{\circ}$. The total energy of the dislocation is

$$
U_{\mathrm{tot}}=U_{\mathrm{I}}+U_{\mathrm{II}}+\tilde{U}
$$

Here, $U_{\mathrm{I}}$ and $U_{\mathrm{II}}$ are the energies associated with the dislocation spread on planes I and II, respectively, and $\tilde{U}$ represents the elastic interaction energy between the dislocation densities on planes I and II. The expressions for $U_{\mathrm{I}}$ and $U_{\text {II }}$ are identical to that given earlier for the single glide plane case, while the new term $\tilde{U}$ can be derived from Nabarro's equation for general parallel dislocations [5]:

$$
\begin{aligned}
U_{\mathrm{I}(\mathrm{II})}= & \sum_{i, j} \frac{1}{2} \chi_{i j}\left\{K_{e}\left[\rho_{1}^{\mathrm{I}(\mathrm{II})}(i) \rho_{1}^{\mathrm{I}(\mathrm{II})}(j)+\rho_{2}^{\mathrm{I}(\mathrm{II})}(i) \rho_{2}^{\mathrm{I}(\mathrm{II})}(j)\right]\right. \\
& \left.+K_{s} \rho_{3}^{\mathrm{I}(\mathrm{II})}(i) \rho_{3}^{\mathrm{I}(\mathrm{II})}(j)\right\}+\sum_{i} \Delta x \gamma_{3}\left(\delta_{1}^{\mathrm{I}(\mathrm{II})}(i), \delta_{2}^{\mathrm{I}(\mathrm{II})}(i), \delta_{3}^{\mathrm{I}(\mathrm{II})}(i)\right) \\
& -\sum_{i, l} \frac{x(i)^{2}-x(i-1)^{2}}{2} \rho_{l}^{\mathrm{I}(\mathrm{II})}(i) \tau_{l}^{\mathrm{I}(\mathrm{II})}+K b^{2} \ln L,
\end{aligned}
$$




$$
\begin{aligned}
\tilde{U}= & -\sum_{i, j} K_{s} \rho_{3}^{\mathrm{I}}(i) \rho_{3}^{p}(j) A_{i j}-\sum_{i, j} K_{e}\left[\rho_{1}^{\mathrm{I}}(i) \rho_{1}^{p}(j)\right. \\
& \left.+\rho_{2}^{\mathrm{I}}(i) \rho_{2}^{p}(j)\right] A_{i j}-\sum_{i, j} K_{e}\left[\rho_{2}^{\mathrm{I}}(i) \rho_{2}^{p}(j) B_{i j}+\rho_{1}^{\mathrm{I}}(i) \rho_{1}^{p}(j) C_{i j}\right. \\
& \left.-\rho_{2}^{\mathrm{I}}(i) \rho_{1}^{p}(j) D_{i j}-\rho_{1}^{\mathrm{I}}(i) \rho_{2}^{p}(j) D_{i j}\right] .
\end{aligned}
$$

Here, $\delta_{1}^{\mathrm{I}(\mathrm{II})}(i), \delta_{2}^{\mathrm{I}(\mathrm{II})}(i)$, and $\delta_{3}^{\mathrm{I}(\mathrm{II})}(i)$ represent the edge, vertical, and screw component of the general dislocation slip vector at the $i$ th nodal point in plane I (II), respectively, while the corresponding component of dislocation density in plane I (II) is defined as before in the planar case. The projected dislocation density $\rho^{p}(\mathrm{i})$ is the projection of the density $\rho^{\mathrm{II}}(\mathrm{i})$ from plane II onto plane I in order to deal with the nonparallel components of the slip vector. $\chi_{i j}, A_{i j}, B_{i j}$, $C_{i j}$, and $D_{i j}$ are double-integral kernels defined by

$$
\begin{aligned}
& \chi_{i j}=\int_{x_{j-1}}^{x_{j}} \int_{x_{i-1}}^{x_{i}} \ln \left|x-x^{\prime}\right| \mathrm{d} x \mathrm{~d} x^{\prime}, \\
& A_{i j}=\int_{x_{j-1}^{\prime}}^{x_{j}^{\prime}} \int_{x_{i-1}}^{x_{i}} \frac{1}{2} \ln \left(x_{0}^{2}+y_{0}^{2}\right) \mathrm{d} x \mathrm{~d} x^{\prime}, \\
& B_{i j}=\int_{x_{j-1}^{\prime}}^{x_{j}^{\prime}} \int_{x_{i-1}}^{x_{i}} \ln \frac{x_{0}^{2}}{x_{0}^{2}+y_{0}^{2}} \mathrm{~d} x \mathrm{~d} x^{\prime}, \\
& C_{i j}=\int_{x_{j-1}^{\prime}}^{x_{j}^{\prime}} \int_{x_{i-1}}^{x_{i}} \ln \frac{y_{0}^{2}}{x_{0}^{2}+y_{0}^{2}} \mathrm{~d} x \mathrm{~d} x^{\prime}, \\
& D_{i j}=\int_{x_{j-1}^{\prime}}^{x_{i}^{\prime}} \int_{x_{i-1}}^{x_{i}} \ln \frac{x_{0} y_{0}}{x_{0}^{2}+y_{0}^{2}} \mathrm{~d} x \mathrm{~d} x^{\prime},
\end{aligned}
$$

where $x_{0}=L-x+x^{\prime} \cos \theta$, and $y_{0}=-x^{\prime} \sin \theta$. The equilibrium structure of the dislocation is again determined by minimizing the total dislocation energy functional with respect to the dislocation density.

\section{Dislocation Core Properties in Aluminum}

The GSF energy surface, $\gamma_{3}\left(\delta_{i}\right)$ entering the P-N model can usually be determined from $a b$ initio calculations based on the density functional theory 
[15]. In Fig. 4, we show the GSF energy surface for $\mathrm{Al}$ which was computed by using a pseudo-potential plane-wave method. The computational detail can be found in [16]. As shown in Fig. 4, the calculated GSF energy surface maintains the underlying translational and rotational symmetry of the fcc lattice. The three high peaks of the GSF surface correspond to the run-on stacking fault configuration $\mathrm{ABC} \mid \mathrm{CABC}$, in which two $\mathrm{C}$ layers are nearest neighbors. The local minimum and maximum along the [112] direction correspond to intrinsic and unstable stacking faults, respectively.

We first examine the core properties of four typical dislocations, i.e., the screw, $30^{\circ}, 60^{\circ}$ and edge dislocations. These dislocations have the same Burgers vector, $\mathbf{b}=\mathrm{a} / 2$ [101], but different orientations (characters).

The results for the energetics and the Peierls stress for the four dislocations are presented in Table 1, along with the values of $\zeta$. First one can see the trend that the half-width $\zeta$ increases monotonically with the dislocation angle $\theta$. Secondly, the misfit energy, $\mathrm{U}_{\text {misfit }}$, also increases monotonically from the screw to the edge dislocation, while the configuration-dependent elastic energy, $U_{\text {elastic }}$ (negative in sign) decreases as the angle increases.

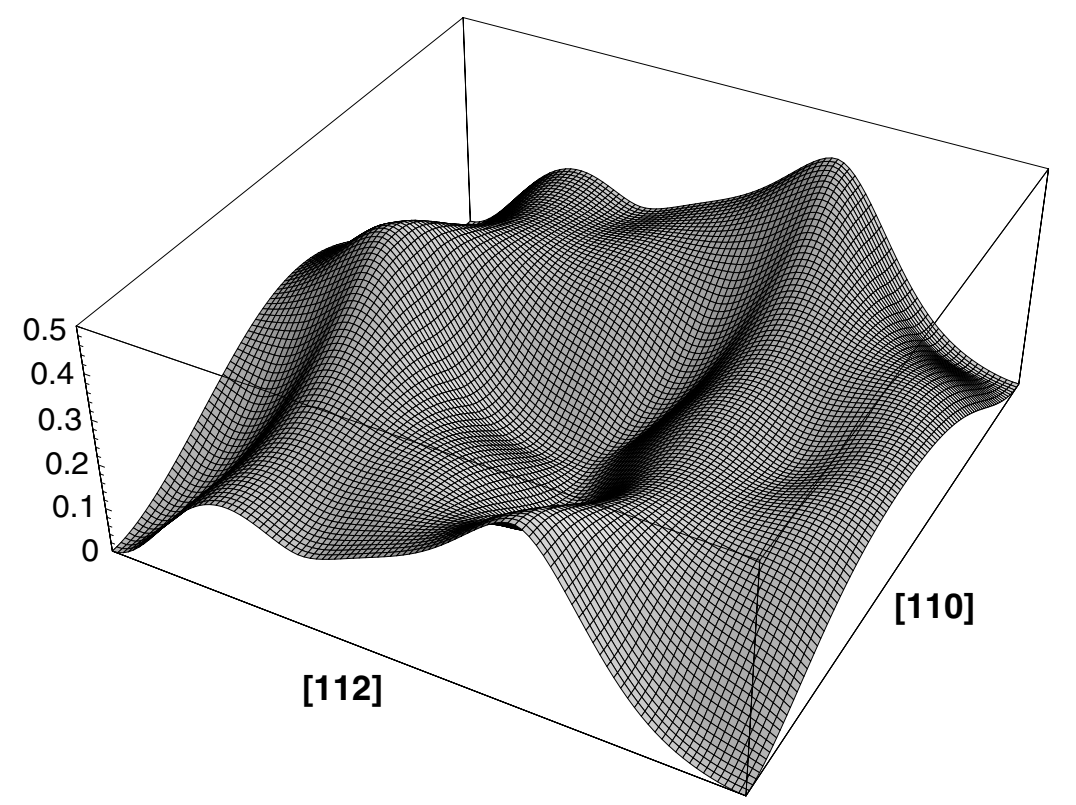

Figure 4. The GSF energy surface for displacements along a (111) plane in $\mathrm{Al}\left(\mathrm{J} / \mathrm{m}^{2}\right)$ (the corners of the plane and its center correspond to identical equilibrium configurations, i.e., the ideal Al lattice) from DFT calculations. 
Table 1. Core half-widths $\zeta$ (in $\AA$ ); core energies $U_{\text {core }}$ and separate contributions from the configuration-dependent elastic energy, $U_{\text {elastic }}$ and the misfit energy $U_{\text {misfit }} ; K b^{2} \ln L$ (in eV/A); and Peierls stress (in MPa) for the four dislocations.

\begin{tabular}{lcccc}
\hline & Screw & $30^{\circ}$ & $60^{\circ}$ & Edge \\
\hline Core widths & 2.1 & 2.5 & 3.0 & 3.5 \\
$U_{\text {core }}$ & -0.0834 & -0.1096 & -0.1678 & -0.1979 \\
$U_{\text {elastic }}$ & -0.1828 & -0.2317 & -0.3199 & -0.3666 \\
$U_{\text {misfit }}$ & 0.0938 & 0.1221 & 0.1521 & 0.1688 \\
$K b^{2} \ln L$ & 1.6050 & 1.8123 & 2.233 & 2.446 \\
Peierls stress & 256 & 53 & 98 & 35 \\
\hline
\end{tabular}

The configuration-independent elastic energy $K b^{2} \ln L$ is also included. Several points need to be emphasized:

(1) The configuration-dependent elastic energy $U_{\text {elastic }}$, ignored in some previous studies, is the dominant contribution to the core energy $U_{\text {core }}$ (about a factor of two larger than $U_{\text {misfit }}$ ). More importantly, it depends strongly on the dislocation character;

(2) While $U_{\text {elastic }}$ is negative here, in principle, it can be of either sign. For example, $U_{\text {elastic }}$ was found to be positive in $\mathrm{Si}$;

(3) Inclusion of the configuration-independent elastic term, $K b^{2} \ln L$, yields positive values for both the total energy and the total elastic energy.

As alluded earlier, the Peierls stress in this work is calculated as the critical value of the applied stress $\tau$, at which the dislocation energy functional fails to be minimized with respect to $\rho_{i}$ through standard conjugate gradient techniques. This approach is more accurate and physically transparent, because it captures the nature of the Peierls stress as the stress at which the displacement field of the dislocation undergoes a discontinuous transition. A typical value for the Peierls stress of $\mathrm{Al}$ from the analysis of the Bordoni internal peaks is about $230 \mathrm{MPa}$, which is very close to our value for the screw dislocation (256 MPa) [17].

In order to correlate dislocation properties with the dislocation character, we have studied dislocation properties of 19 different dislocations that have the same Burgers vector but different orientations. The angle between the dislocation line and the Burgers vector varies from $0^{\circ}$ to $90^{\circ}$. The core energy, along with its separate contributions from the configuration-dependent elastic energy $U_{\text {elastic }}$ and the misfit energy $U_{\text {misfit }}$, are presented in Fig. 5 as a function of the dislocation angle $\theta$. We find that $U_{\text {core }}$ and $U_{\text {elastic }}$ decrease monotonically as the angle increases, whereas $U_{\text {misfit }}$ increases with $\theta$. The configuration-dependent elastic energy $U_{\text {elastic }}$ decreases with $\theta$ because the prelogarithmic factor $K$ increases with $\theta$. On the other hand, the monotonic increase of $U_{\text {misfit }}$ with $\theta$ is due to the fact that the core width increases with 


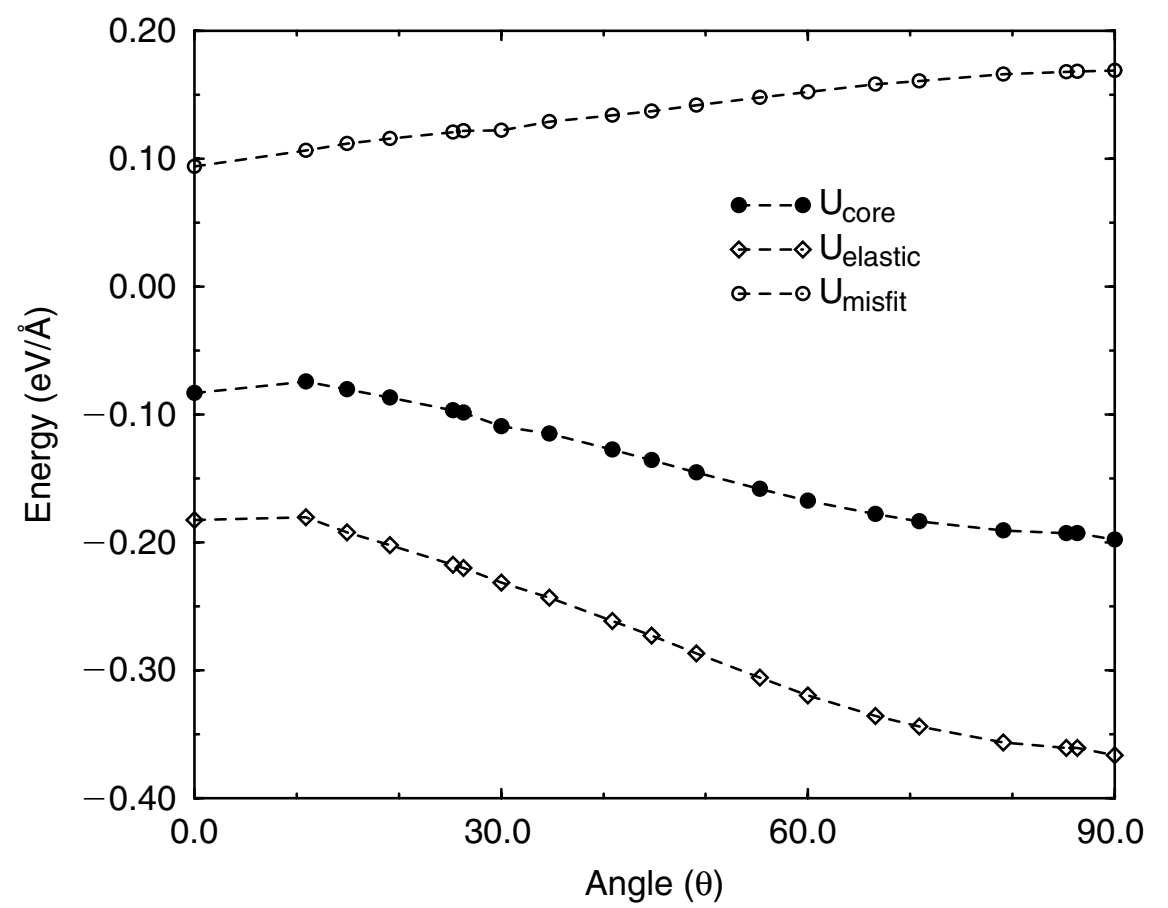

Figure 5. The core energy, elastic energy and misfit energy as a function of dislocation orientations.

the dislocation angle. Note that the configuration-dependent elastic energy, not only is the dominant contribution to the total energy stored in the core region, but also is more sensitive to the dislocation character than the misfit energy.

To correlate the Peierls stress with the dislocation character, we plotted $\ln \left(\sigma_{p} \bar{a} / K b\right)$ as a function of $\zeta / \bar{a}$ in Fig. 6. Here, $\zeta$ is the half-width of a dislocation and $\bar{a}$ is the average nodal spacing along the $x$ direction. It should be pointed out that most of the dislocations in the fcc lattice have noneven nodal spacing, except for the $30^{\circ}$ and edge dislocations. Most of the calculated values can be fitted (solid line) with

$$
\sigma_{p}=\frac{2 \pi K b}{\bar{a}} e^{-1.7 \zeta / \bar{a}}
$$

The large deviation of $\sigma_{P}$ for the $30^{\circ}$ and edge dislocations from the common trend, indicates that the nodal spacing (even vs. non-even) between atomic planes plays an important role on the Peierls stress [18]. On the other hand, the deviation of the $10.9^{\circ}$ and $14.9^{\circ}$ dislocations from the common trend is 


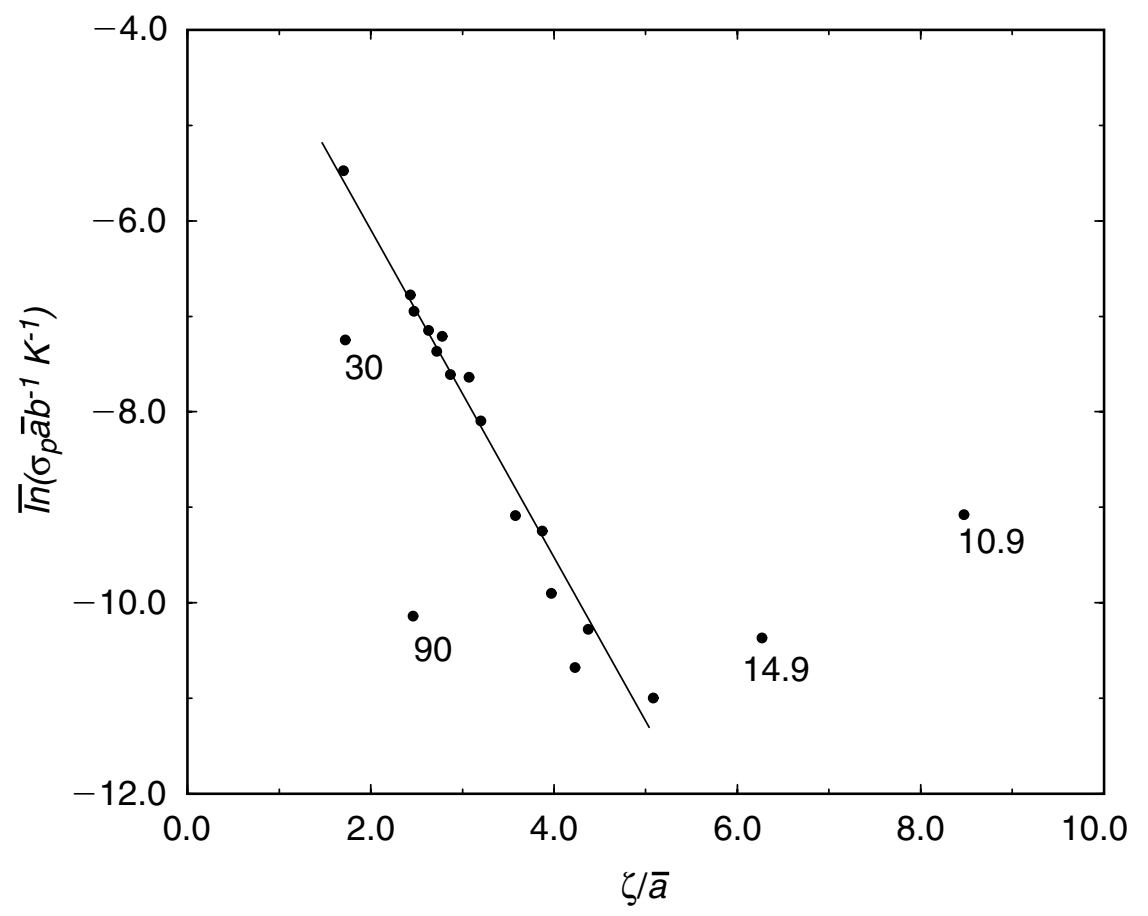

Figure 6. The scaled Peierls stress as a function of the ratio of the core width to the average atomic spacing perpendicular to the dislocation line.

unclear to us at present. Note, that the Peierls stress is more sensitive to the average atomic spacing $\bar{a}$ than to the half-width. For example, while both the $0^{\circ}$ and $14.9^{\circ}$ dislocations have predominant screw components and similar half-widths of $2.1 \AA$ and $2.3 \AA$, respectively, they have quite different atomic spacings, $1.2 \AA$ and $0.3 \AA$, respectively. This results in a Peierls stress of $6 \mathrm{MPa}$ for the $14.9^{\circ}$ dislocation, almost two orders of magnitude smaller than that of $256 \mathrm{MPa}$ for the screw dislocation.

\section{Conclusion}

To conclude, the $\mathrm{P}-\mathrm{N}$ model serves as a link between atomistic and continuum approaches, by providing a means to incorporate information obtained from atomistic calculations (ab initio or empirical) directly into continuum models. The resultant approach can then be applied to problems that neither atomistic nor conventional continuum models could handle separately. The simplicity of the $\mathrm{P}-\mathrm{N}$ model makes it an attractive alternative to direct 
atomistic simulations of dislocation properties. It provides a rapid and inexpensive route to determine dislocation core structure and mobility. Combined with $a b$ initio determined GSF energy surface, the $\mathrm{P}-\mathrm{N}$ model could give rather reliable quantitative predictions for various dislocation properties. Furthermore, since $a b$ initio based $\mathrm{P}-\mathrm{N}$ model calculations are much more expedient than direct $a b$ initio atomistic calculations for dislocations, the $\mathrm{P}-\mathrm{N}$ model could serve as a powerful and efficient tool for alloy design, where the goal is to select the "right" elements with the "right" alloy composition to tailor desired mechanical, and in particular, dislocation properties. Finally, we should comment that the $\mathrm{P}-\mathrm{N}$ model is just one example of more general cohesive surface models that are built upon the idea of limiting all constitutive nonlinearity to certain privileged interfaces, while the remainder of materials is treated through more conventional continuum theories. The same strategy has also been applied to the study of fracture and dislocation nucleation from a crack tip [19].

\section{References}

[1] M.S. Duesbery, "Dislocation core and plasticity," Dislocations in Solids, F.N.R. Nabarro, ed., vol. 8, 67, North-Holland, Amsterdam, 1989.

[2] M.S. Duesbery and G.Y. Richardson, "The dislocation core in crystalline materials," CRC Crit. Rev. Sol. State Mater. Sci., 17, 1, 1991.

[3] V. Vitek, "Structure of dislocation cores in metallic materials and its impact on their plastic behavior," Prog. Mater. Sci., 36, 1, 1992.

[4] R. Peierls, "The size of a dislocation," Proc. Phys. Soc. London, 52, 34, 1940.

[5] F.R.N. Nabarro, "Dislocations in a simple cubic lattice," Proc. Phys. Soc. London, 59, 256, 1947.

[6] J.D. Eshelby, "Edge dislocations in anisotropic materials," Phil. Mag., 40, 903, 1949.

[7] V. Vitek, "Intrinsic stacking faults in body-centered cubic crystals," Phil. Mag., 18, 773, 1968.

[8] J.P. Hirth and J. Lothe, Theory of Dislocations, 2nd edn., Wiley, New York, 1992.

[9] G. Schoeck, "The core energy of dislocations," Acta Metall. Mater., 127, 3679, 1995.

[10] J.W. Christian and V. Vitek, "Dislocations and stacking faults," Rep. Prog. Phys., 33, 307, 1970.

[11] J. Wang, "A new modification of the formulation of peierls stress," Acta Mater, 44, 1541, 1996.

[12] G. Schoeck, "Peierls energy of dislocations: a critical assessment", Phys. Rev. Lett., 82, 2310, 1999.

[13] V. Bulatov and E. Kaxiras, "Semidiscrete variational peierls framework for dislocation core properties," Phys. Rev. Lett., 78, 4221, 1997.

[14] G. Lu, V. Bulatov, and N. Kioussis, "A non-planar peierls-nabarro model and its application to dislocation cross-slip," Phil. Mag., 83, 3539, 2003.

[15] P. Hohenberg and W. Kohn, "Inhomogeneous electron gas," Phys, Rev., 136, B864, 1964. 
[16] G. Lu, N. Kioussis, V. Bulatov, and E. Kaxiras, "Generalized-stacking-fault energy surface and dislocation properties of aluminum," Phys. Rev. B, 62, 3099, 2000a.

[17] W. Benoit, N. Bujard, and G. Gremaud, "Kink dynamics in f.c.c. metals," Phys. Stat. Sol., (a), 104, 427, 1987.

[18] G. Lu, N. Kioussis, V. Bulatov, and E. Kaxiras, "The peierls-nabarro model revisited," Phil. Mag. Lett., 80, 675, 2000b.

[19] J.R. Rice, "Dislocation nucleation from a crack tip: an analysis based on the peierls concept," J. Mech. Phys. Sol., 40, 239, 1992. 